\title{
Improvement and simulation of ZRP routing protocol based on Beidou Satellite
}

\author{
Tang Qing ${ }^{1, ~ a ~}$, Bo Yang ${ }^{1, a}$, Lv Liangshuang ${ }^{1, a}$, Xia Chunhe ${ }^{1, \text { a }}$ \\ ${ }^{1}$ Beijing Key Laboratory of Network Technology, Beihang University, Beijing, 100191, China \\ aemail: tangqing_bj@163.com
}

\begin{abstract}
Keywords: The Wireless Ad Hoc Network; ZRP Routing Protocol; Beidou Satellite; The Control Center
\end{abstract}

\begin{abstract}
In this article, Wireless Ad hoc Networks and routing classification are briefly presented and ZRP Routing Protocols are analyzed. In large wireless Ad hoc networks, ZRP routing protocol takes long time to find routing path. It puts forward the improved routing protocol IZRP. This protocol uses the Beidou satellite to transmit route request and uses the control center to calculate the routing path. At last, this article verity the ZRP Routing Protocol and IZRP Routing Protocol using the software platform of Opnet Modeler and get the conclusion that under the condition of more nodes , IZRP routing protocol routing time delay is short, high efficiency.
\end{abstract}

\section{Introduction}

The mobile Ad Hoc network is a temporary self-healing system that needs no fixed base installations and is composed of several wireless mobile devices. Unlike the traditional network, the nodes inside Ad Hoc network moving randomly and frequently coming in and going out of the network, result in the constant changes of the topological structure of the network, and decide that each node inside the network proceeds multihop communication and exchange information through the wireless link. At the present, the wireless Ad Hoc network is widely used, and it can be used as both the independent network and the expended network of the wired network. The independent mobile Ad Hoc network is mainly used in the military field, and in the modernized and paroxysmal military battlefields, the communication is almost impossible like installing telegraphs in the 60s, and the Ad Hoc network which has powerful self-healing effect and destroy-resistance becomes a good choice. As the expended network of the existing wired network, Ad Hoc network, just as its name implies can be installed in the zones that the wired network can be installed, such as emergency aid disaster releasing, sensor system.

Ad Hoc network has many routing protocols, which can be divided into the following categories [2]: according to the differences of the topological structures, divided into plane routing protocol and layered routing protocol; according to the routing dynamics, divided into the active routing protocol, on-demand routing protocol, and hybrid routing protocol; according to the difference of the destination node numbers, divided into unicast routing [3] protocol and multicast routing protocol. And influenced by the traditional network, the most discussed is the routing protocol divided according to the routing dynamics.

The active routing protocol is also called the instrument-driven routing protocol or proactive routing protocol, and each node inside the network will regularly proceed the route discovery to guarantee that the routing tables inside the nodes can maintain the topology of the network in real time, for instance, the protocols of DSDV[4], OLSR[5], STAR, etc. the on-demand rotung protocol is also called the reactive rotuing protocol, and the nodes inside the network will proceed the arithmetic of rotue discovery only under the need of sending the data, and there is no need to maintain the topology of the whole network, for instance, DSR[7], AODV[8] , etc. The extreme conditions of active rotuing protocol and on-demand rotuing protocol are just suitable for some specified Ad Hoc networks, and the active rotuing protocol requires network information exchanges in real time, and the performances of the small-size network are excellent, but it is fatefully not qualified to the large-size network. The on-menad rotuing protocol needs no maintenance of the 
network topology in real time, which proves that it can be used in the large-size network, but its performances in the small-size network are inferior to thoses of the active rotuing protocol. And presentation of the hybrid rotue, however tactically merges the advanteges of both together.

\section{Routing Protocol}

\section{$\mathrm{ZRP}$ routing zones.}

In the ZRP (Zone Routing Protocol), set some node as I, and n represents the zone radius, $\mathrm{H}$ (i,j) means the shortest hop count from node $\mathrm{j}$ to node $\mathrm{i}$. Then the routing zone of node I is:

$$
\mathrm{Z}(n, i)=\{j \mid H(i, j) \leq n\}
$$

ZRP routing protocol stipulates that the active routing protocol shall be used within the zone radius, and on-demand routing protocol shall be used outside the zone, and its performances are decided by the parameter values of the zone radius to a great extent. In the Ad Hoc network having $\mathrm{N}$ nodes, ZRP protocol regulates that each node, taking itself as the center, hop count as the radius, divides the whole network into $\mathrm{N}$ overlapping zones.

\section{Components of ZRP protocol.}

IARP [10], IERP and BRP together compose the entity of ZRP protocol. IARP is the intra-zone routing protocol, as its name implies, it is a small-size active routing protocol with limited hop count. And its basic function is neighbor discovery, and it can directly get or adopt the neighbor discovery protocol NDP through MAC layer, that is to regularly broadcast the Hello message to collect information, and IARP saves the routing information of all nodes within this zone.

IERP is the sub-protocol of the intra-zone on-demand protocol. And when the nodes using the IARP protocol find that the destination nodes are outside the zone scope, IERP will through the border multicast routing to request to traverse the network and search for the destination nodes.

The presentation of BRP protocol is to reduce the routing redundancy or repeated broadcastings, in order to improve the efficiency of routing requiring the broadcast in IERP.

\section{ZRP Routing Protocol Based on the Beidou Satellites Navigation System}

\section{Defects of ZRP routing protocol.}

Although ZRP protocol combines the advantages of the active routing protocol and on-demand routing protocol, the active routing protocol is used within the zone scope, and the size of the routing zone radius can be defined according to the actual physical environment. But the defects of ZRP protocol lie in: for instance, under some complicated environments, the zone routing radius shall be reduced in order to guarantee the routing speed within the zone, and this must result in the rising intra-zone routing delay. Or when the wireless ad hoc network is relatively large, the zones will be overmuch, and this will also result in the fast rising of the intra-zone routing delay.

\section{The improved ZRP routing protocol.}

The improved routing protocol is temporarily called IZRP, (Improved Zone Routing Protocol, and hereafter called IZRP), and its purpose is: aiming at the intra-zone routing protocol of the ZRP routing protocol to provide a fast routing method based on the Beidou Satellites Navigation System. And this method has the characteristics of fast route discovery, simple route maintenance, and fast convergence, etc.

The IZRP routing protocol basis based on the Beidou Satellites Navigation System is composed of three large modules (see Figure 1): wireless ad hoc network, Beidou Satellites Navigation System, and the control center of the ad hoc network. The Beidou Satellites provides the services of localization and short message communication to the wireless nodes. And the control center receives the routing request, and provides functions of route discovery and route maintenance to the ad hoc network. 


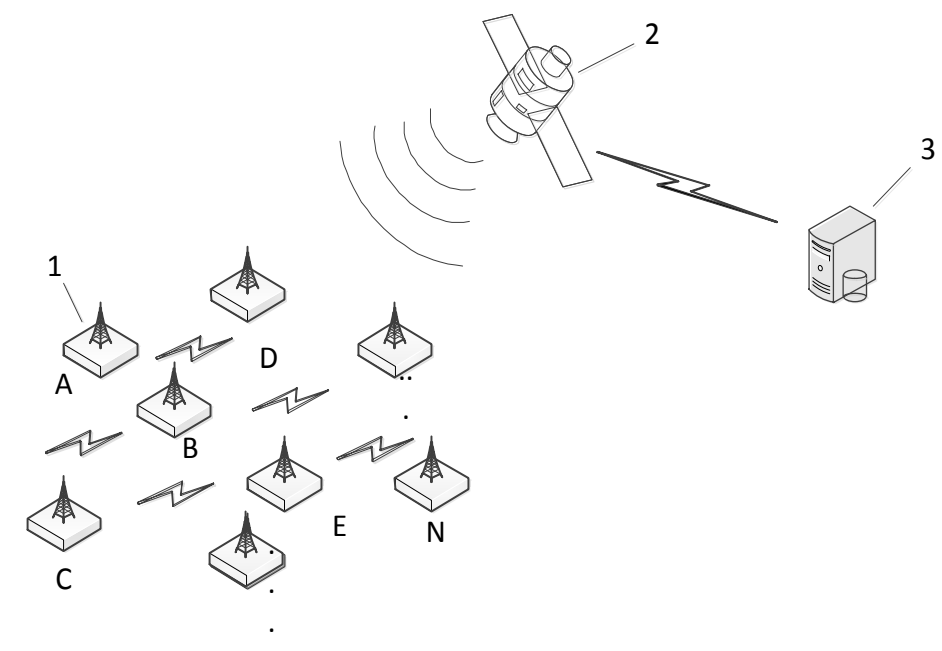

Fig.1. IZRP routing protocol module

See Figure 2, the working processes of the IZRP routing protocol are:

Step 1 each node regularly receives the localization signals of Beidou Satellites for localization, and scan and record the surrounding neighbor nodes.

Step 2 each node, through the function of short message communication of Beidou Satellites, sends its own number, geographical coordinates and neighbor nodes to the control center.

Step 3 when the original node sends the communication request to the destination node, first of all, use the active routing protocol IARP to find if the destination node is within its own zone scope, and if it is within the zone scope, then communicate directly.

Step 4 if the destination node is outside the zone scope, then the original node shall send the routing request of connecting the destination node through the function of short message communication of the Beidou Satellites.

Step 5 after the control center receives the routing request of the original node, it shall search for the coordinates of the destination node, and calculate the route from the original to the destination node.

Step 6 send the routing request of the original node and the routing route to each node on the links through the Beidou Satellites.

Step 7 after the destination node receives the routing request, according to the route, it shall directly reply the confirmation message to the original node through the ad hoc network.

Step 8 after the original node receives the confirmation message, then it means the routing route is clear to start the communication.

Step 9 if the original node fails to receive the confirmation message from the destination node within the specific time, then it shall send the routing request again through the Beidou Satellites. 


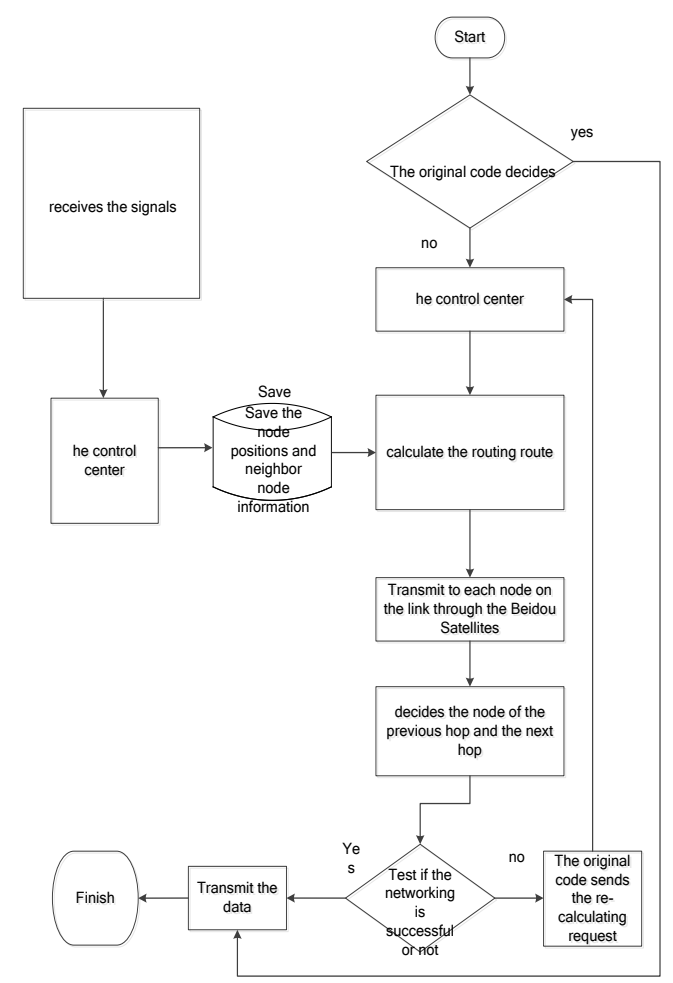

Fig.2. Working processes of the IZRP routing protocol

\section{Experiment}

\section{Simulation environment.}

The experiment adopts Opnet Modeler 14.5 to carry out simulation on the mobile Ad hoc network. And in this experiment, the routing protocols are separately adopted with ZRP and IZRP, and it gives the simulation comparative results of the route discovery delay by means of figures at last.

Within the $2000 \mathrm{~m} \times 2000 \mathrm{~m}$ plane square zone of the simulation scene, set up different network nodes according to the different network scale settings, which are separately 50、100、200、 400、800、1000、1200. In order to objectively compare the performances of the routing protocols, it is set to start one route discovery every one second in the experiment, and successively record the route discovery delay. Compare the ZRP routing protocol and IZRP protocol by comparing the route discovery delays of different scales.

In each simulation experiment, the node is separately adopted with the half numbers of the nodes of powerful mobility (moving speed $20 \mathrm{~m} / \mathrm{s} \sim 30 \mathrm{~m} / \mathrm{s}$ ), and half number of the nodes of weak mobility (moving speed $0 \mathrm{~m} / \mathrm{s} \sim 10 \mathrm{~m} / \mathrm{s}$ ), and the moving model is adopted with the Random Waypoint model. The MAC layer is adopted with the distributed coordination function, DCF[13] stipulated by IEEE802.11b to resolve the problem of hidden terminal[14]. The routing radius is 3, and OSI model within the node is as shown in Figure 3. 


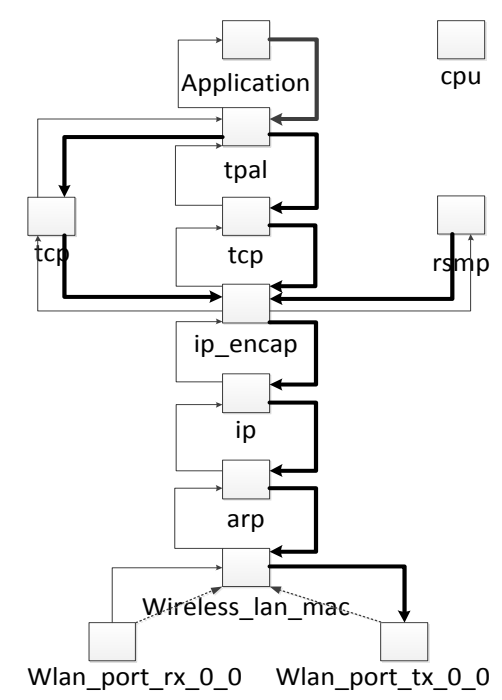

Fig.3. Node OSI model set

Because in the routing outside the zone, the routing request is transmitted by the Beidou Satellites to the control center, and the simulation adopts the coordinate style here, and uses the node coordinates to replace the geographical coordinates. Taking the actual conditions into consideration, the height of the Beidou Satellites is about $35883 \mathrm{~km}$, and the time of a round trip for the electromagnetic waves and the control center calculates the routing route is about 0.5 second, which will be directly calculated into the route discovery delay outside the zone.

\section{Simulation results.}

The time difference from the original node sending out the routing request to it receiving one routing is called the route discovery delay. And it reflects the routing efficiency of the routing protocols.

Aiming at different network scales, each experiment adopts different nodes. And in each experiment, the ZRP protocol and IZRP protocol shall be separately operated under the condition of keeping the node numbers and other parameters unchangeable.

Because the experiments have been carried out for many times, here we just give the network experiment map when the node numbers are separately 100 and 900.

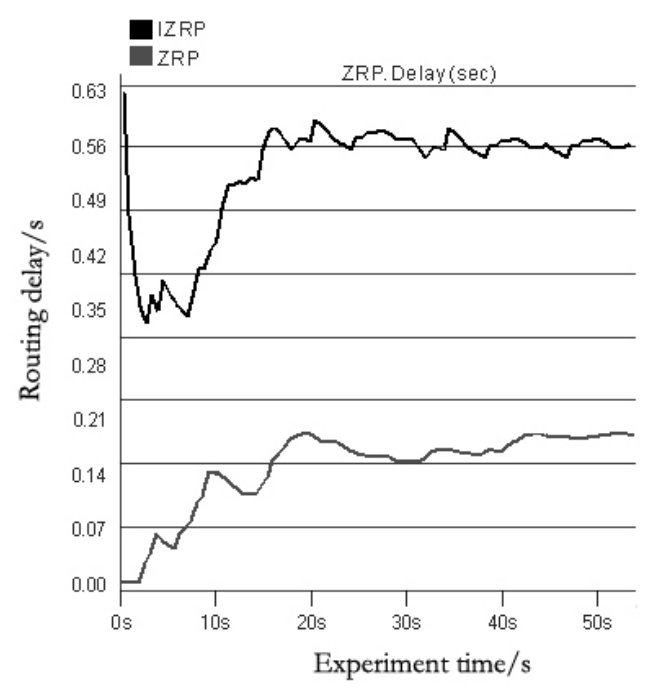

Fig.4. Route discovery delay when the node number is 100 


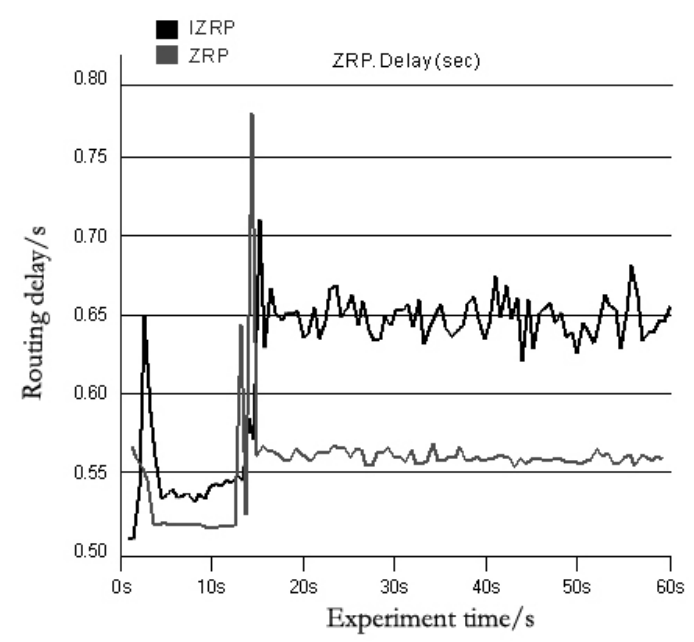

Fig.5. Route discovery delay when the node number is 900

Figure 4 shows the route discovery delay when the node number is 100 , and from this figure it can be seen that the average route discovery delay of ZRP routing protocol is about $0.15 \mathrm{~s}$, and the average route discovery delay of IZRP routing protocol is about $0.55 \mathrm{~s}$. from the data it can be seen that ZRP routing protocol has more advantages than IZRP protocol. Figure 5 shows the route discovery delay when the node number is 900 , and the route discovery delay of ZRP protocol is about $0.66 \mathrm{~s}$, and the route discovery delay of IZRP protocol is about $0.57 \mathrm{~s}$. The experiment data of each experiment are as shown in the following Figure 5.

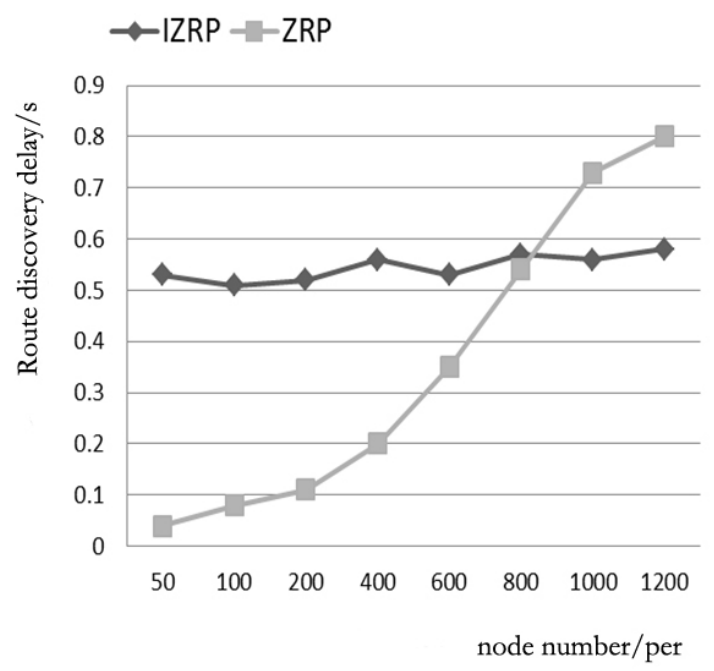

Fig.6. route discovery delay

Figure 6 shows the relationship between the route discovery delay and the node numbers. From this figure it can be seen that when the total numbers of the nodes are relatively small, the average delay of ZRP route discovery is relatively short. And when the node numbers are relatively large, the average route discovery delay increases sharply. When the node number reaches 800 , the route discovery delay of ZRP and IZRP are equivalent to each other under the specific experimental environments. And when the node numbers continuously increase, the advantages of IZRP routing protocol will gradually appear. That is because for the small-size ad hoc network, there are relatively less node transmission routing requests outside the zone hence the route discovery delay of IZRP will be relatively short. Relatively speaking, for the large-size ad hoc network, the routing requests must be transmitted for many times to find the destination node; hence the route discovery delay of ZRP will be relatively long. While IZRP routing protocol transmits the routing request by the satellites, the control center will directly calculate the routing route according to the geographical positions of the original node and destination node, which saves the time of routing research. 


\section{Conclusion}

ZRP routing protocol combines the advantages of the active routing protocol and on-demand routing protocol, and the efficiency of the routing protocol has been greatly improved, but this advantages are just limited within the small-size wireless ad hoc network. Compared to the wireless as hoc networks of middle or large size, it will improve the routing efficiency to appropriately increase the zone radius, but the effects are limited and can not solve the problem basically. IZRP routing protocol transmits the routing request through the Beidou Satellites, and the routing route is calculated by the control center, which effectively solve the route discovery efficiency problem of the ad hoc networks of middle and large sizes.

\section{Acknowledgement}

National science foundation of china No 61170295

The project is supported by the national ministries and commissions A2120110006

\section{References}

[1] Perkins.C.E and B.hagwat P.Highly dynamic destination-sequenced distance vector routing (DSDV) for mobile computers[C]. Comp Comm Rev,1994:234-244.

[2] Istikmal. Analysis And Evaluation Optimization Dynamic Source Routing ( DSR ) Protocol in Mobile Ad hoc Network Based on Ant Algorithm[J]. International Conference of Information and Communication Technology (ICoICT), 2013, 5(13):400-404.

[3] Zhong, Chen, Stefan Müller Arisona, Xianfeng Huang, Michael Batty, and Gerhard Schmitt. Detecting the dynamics of urban structure through spatial network analysis. International Journal of Geographical Information Science 28, no. 11 (2014): 2178-2199.

[5] Li, Wubin, Johan Tordsson, and Erik Elmroth. An aspect-oriented approach to consistency-preserving caching and compression of web service response messages. In Web Services (ICWS), 2010 IEEE International Conference on, pp. 526-533. IEEE, 201

[6] Y. Geng, J. He, K. Pahlavan, Modeling the Effect of Human Body on TOA Based Indoor Human Tracking[J], International Journal of Wireless Information Networks 20(4), 306-317

[7] Lv, Zhihan, and Tianyun Su. 3D seabed modeling and visualization on ubiquitous context. In SIGGRAPH Asia 2014 Posters, p. 33. ACM, 2014. 